\title{
Evidence for crystal-field splitting in surface-atom photoemission from potassium
}

\author{
G. K. Wertheim \\ AT\&T Bell Laboratories, Murray Hill, New Jersey 07974-0636 \\ D. M. Riffe \\ Department of Physics, Utah State University, Logan, Utah 84322-4415
}

(Received 15 August 1995)

\begin{abstract}
Photoemission spectra from the shallow $3 p_{3 / 2}$ core levels of the surface atoms of metallic potassium exhibit the effects of a small but measurable crystal-field splitting of $\sim 38 \mathrm{meV}$. It manifests itself mainly as an apparent angle-dependent modulation of the spin-orbit splitting. This phenomenon may, in general, interfere with accurate determinations of surface-atom core-level shifts.
\end{abstract}

Photoemission has demonstrated that the perturbation of the band structure at the surface of most metals is largely confined to the outermost atomic layer. The evidence lies in the finding that only the surface layer of atoms exhibit measurably different core-electron binding energies, ${ }^{1}$ larger ${ }^{2}$ and anharmonic ${ }^{3}$ phonon broadening, as well as different singularity indices ${ }^{4}$ and core-hole lifetimes. ${ }^{5}$ Smaller subsurface shifts have been identified in only a few metals. ${ }^{6-8}$ The surface-atom core-level shift has been well documented experimentally for many different types of metal, and is in agreement with various theoretical approaches. ${ }^{9,10}$

Another expected phenomenon, namely the crystalfield splitting of the core-level spectrum from surface atoms of a bulk metal, has not been observed. It has, however, been reported for atoms adsorbed on a foreign substrate, ${ }^{11,12}$ suggesting that a more careful investigation of bulk metals is warranted. Even if the splitting itself is too small to be resolved, it may still be manifest in changes with takeoff angle in the photoemission spectrum from a single crystal. For small perturbations, the major effect of the crystal field on a $p$ level is to split the $p_{3 / 2}$ line into two components with distinct angle-dependent intensities. ${ }^{13}$ Since the sum of these intensities is a constant (relative to the $p_{1 / 2}$ feature), the net effect of a small, unresolved crystal-field splitting is an angledependent modulation of the separation of the spin-orbit (SO) components.

We have carried out experiments with $\mathbf{K}$ grown from the vapor phase on a $\mathrm{Ni}(100)$ substate at liquid-nitrogen temperature to search for this effect. Since bcc K grows with a (110) orientation on the (100) surface of fcc $\mathrm{Ni}^{2,14}$ the surface atoms occupy sites with twofold symmetry. Photoemission data were taken on beam line U4A at the National Synchrotron Light Source, which is equipped with a 6-m toroidal grating monochromator and a 100$\mathrm{mm}$ hemispherical electron energy analyzer. In the experiment the incident light beam is plane polarized with the $E$ field at a constant $45^{\circ}$ with respect to the electronemission direction. Angle-dependent data were obtained by rotating the sample normal in a plane defined by the polarization vector and the emission direction. In the data discussed below, a takeoff angle of $45^{\circ}$ corresponds to radiation incident approximately normal to the sample surface, while $-40^{\circ}$ corresponds to radiation near grazing incidence. We confine the investigation to relatively large photon energies in order to minimize the effects of structure in the unoccupied conduction band. In particular, we exclude the region within $10 \mathrm{eV}$ of the Fermi level, where resonant excitation involving the empty $3 d$ bands has been shown to have a strong influence on valence-band photoemission. ${ }^{15}$

A set of takeoff-angle-dependent spectra obtained with 45-eV radiation are shown in Fig. 1. The data consist of overlapping bulk and surface doublets, as indicated at the bottom of the figure. The spectra are shown normalized to give the central peaks the same amplitude. The binding energy of the bulk $3 p_{3 / 2}$ peak has a root-mean-square deviation of $3 \mathrm{meV}$ in the spectra shown, providing a measure of the instrumental stability. The broader surface $3 p_{1 / 2}$ line also has an angle-independent binding energy, but the middle peak exhibits a significant systematic angle-dependence shift. The sign of this shift is consistent with that expected on the basis of the angledependent bulk-to-surface intensity ratio, but is larger than expected. Inspection does not give any indication of a crystal-field splitting of the surface contribution to the central peak. However, since the bulk $3 p_{1 / 2}$ and surface $3 p_{3 / 2}$ components overlap in the central peak, such splitting may well be obscured.

We have explored various methods of separating the bulk and surface contributions in these spectra. The easiest one, which was successfully used in the case of $\mathrm{Na} 2 p$ spectra (see Fig. 1 of Ref. 2 ) is to strip out the $p_{1 / 2}$ components using a simple subtractive method. This approach has the attractive feature that it does not require the use of calculated line shapes, i.e., it is model free. Acceptable results were not obtained when this method was applied to the $\mathbf{K}$ data because two of the assumptions on which this method is predicated are not satisfied: The SO components do not have the same lifetime widths, and the effective SO splitting of the bulk and surface are not identical (see below).

Another model-free approach ${ }^{1}$ is based on the assump- 


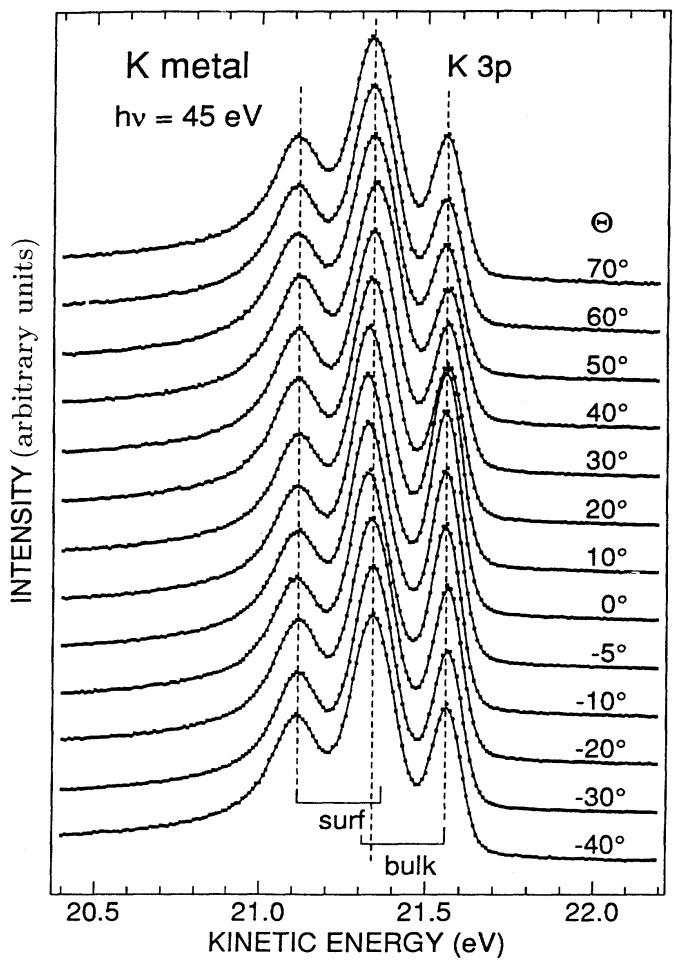

FIG. 1. Spectra of the $\mathrm{K} 3 p$ photoelectrons obtained with 45-eV synchrotron radiation as a function of takeoff angle (measured from the surface normal).

tion that angle-dependent effects are limited to those resulting from the inelastic mean free path of the photoelectrons. Spectra taken with different takeoff angles are then simply linear combinations of unique bulk and surface spectra with different relative amplitudes. Two such spectra suffice to determine the bulk and surface contributions. The results of this procedure are illustrated in Fig. 2, using data taken with $70-\mathrm{eV}$ photons at normal emission $\left(\Theta=0^{\circ}\right)$ and with a $55^{\circ}$ takeoff angle. The combining ratio was empirically adjusted either to minimize the bulk peak at $18.34 \mathrm{eV}$, thereby isolating the surface spectrum, or to minimize the surface peak at $18.79 \mathrm{eV}$, thereby isolating the bulk spectrum. The results show a SO splitting of $\sim 250 \mathrm{meV}$ and a surface-atom core-level shift of $\sim 200 \mathrm{meV}$. They are in reasonable accord with earlier determinations, ${ }^{3,16}$ and confirm that the bulk and surface doublets overlap as shown at the bottom of Fig. 1.

More detailed analysis requires fitting the original data with two overlapping Doniach-Šnjic ${ }^{17}$ SO doublets, each defined by eight parameters: two lifetime widths, two line positions, two amplitudes, one singularity index, and one Gaussian (phonon plus instrumental) width. When all the parameters for each of these two doublets are left free, no useful output is obtained. The problem is due in large part to the fact that there is no unique assignment for the positions and amplitudes of the two unresolved lines that overlap to produce the middle peak, but there are also strong correlations between the width and lineshape parameters of the bulk and surface lines. Physical-

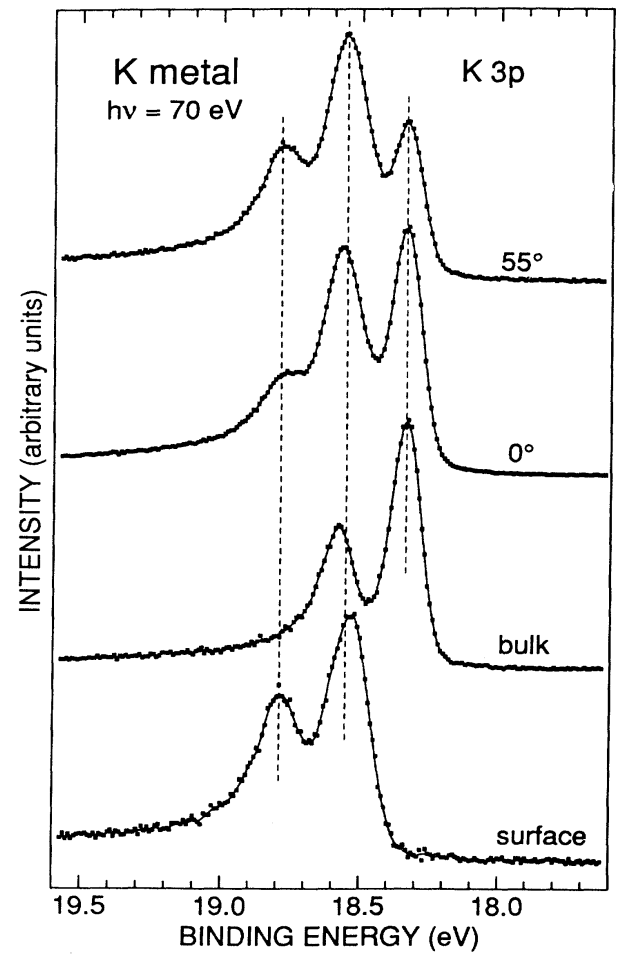

FIG. 2. Spectra of the $\mathrm{K} 3 p$ level obtained with $70-\mathrm{eV}$ radiation at normal emission and $55^{\circ}$ takeoff angle. Also shown are bulk and surface $\mathrm{K} 3 p$ spectra, isolated by taking linear combinations of the above data.

ly motivated constraints are needed to reduce the number of free parameters. ${ }^{16}$ The most useful one is to fix the SO area ratio of the bulk peak at the statistical value of $\frac{1}{2}$. (We note that the SO ratio must be determined from areas rather than peak heights, since the two SO components have different lifetime widths.) It is tempting to assume that the bulk and surface SO splitting are also identical. When both of these constraints are applied to the data in Fig. 2, the $0^{\circ}$ and $55^{\circ}$ data yield significantly different SO splittings, 245 and $257 \mathrm{meV}$, respectively. This indicates that the assumption that the bulk and surface SO splittings are identical must be abandoned.

Since the SO splitting of the bulk spectrum is certainly angle independent, while that of the surface may have an angular dependence due to an unresolved crystal-field splitting, we fix the bulk splitting to a predetermined value and leave the surface value free. The bulk SO splitting is determined from data taken with larger photon energy, where the bulk contribution dominates. Figure 3 displays data taken in normal emission with $115-\mathrm{eV}$ photons. Two SO doublets, representing the bulk and surface, with the bulk SO intensity ratio constrained to the statistical value of $\frac{1}{2}$, provide a satisfactory fit to the data. Data taken with $85-$ and $130-\mathrm{eV}$ radiation give similar results. The bulk SO splitting is $254 \pm 4 \mathrm{meV}$, in reasonable agreement with the value obtained from the bulk spectrum isolated by taking a linear combination of the experimental spectra in Fig. 2. It is also in good agreement 


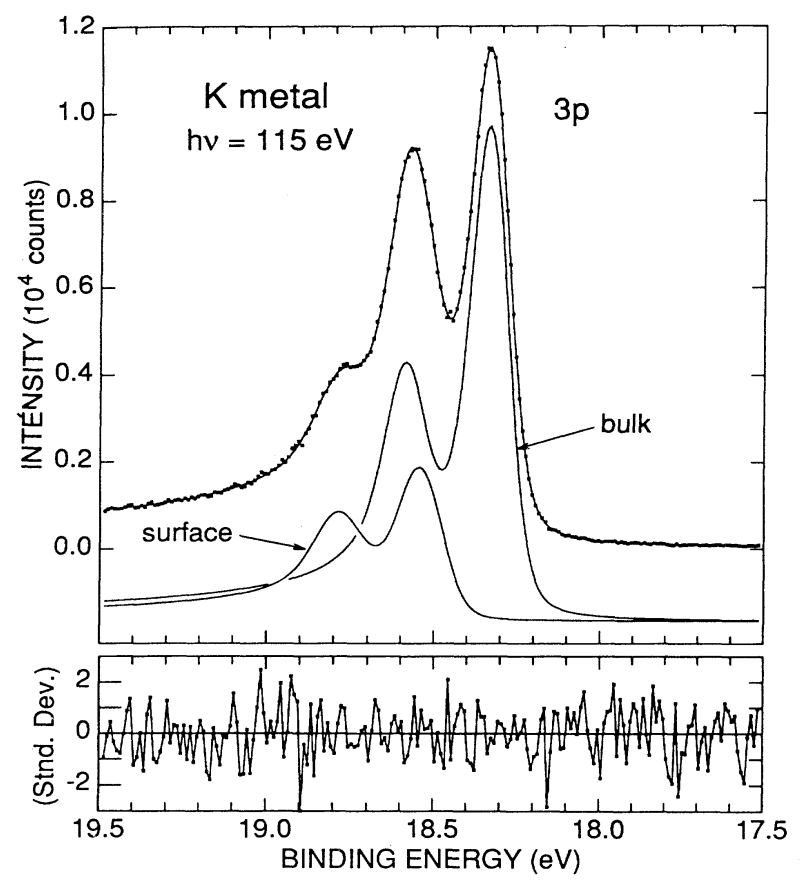

FIG. 3. Fit to $\mathrm{K} 3 p$ spectrum taken at normal emission with $115-\mathrm{eV}$ radiation. The bulk spin-orbit ratio was constrained to the statistical value of $\frac{1}{2}$.

with the value of $257.4 \mathrm{meV}$ obtained from atomic spectra for the $3 p^{5} 4 s^{2}$ configuration, ${ }^{18}$ which corresponds to that of the fully screened hole state in the metal. The bulk singularity index obtained from this data set is $0.14 \pm 0.01$, and the surface-atom core-level shift is 197 $\mathrm{meV}$. The error bars correspond to the range of values obtained with different constraints on the surface lifetime width.

In order to obtain reliable values for the effective surface SO splitting of the data in Fig. 1, we proceed as follows: We fit the data with constraints on the bulk SO splitting and ratio, and look for parameters that yield consistent values from all spectra. These are then constrained to their average value, and the process repeated. This is necessary because parameter correlations allow fluctuations in one parameter to affect the value of others. Fixing the bulk SO splitting at $254 \mathrm{meV}$ and the bulk SO ratio at 0.5 , we obtain bulk lifetime widths of $19 \pm 3 \mathrm{meV}$ for $3 p_{3 / 2}$ and $31 \pm 5$ for $3 p_{1 / 2}$, in very good agreement with values obtained from soft-x-ray emission spectra. ${ }^{19}$ The surface lifetime widths are both larger by a factor of 2. The singularity indexes are $0.145 \pm 0.010$ for the bulk and $0.19 \pm 0.02$ for the surface. The surface-atom corelevel shifts, determined from the $p_{1 / 2}$ lines, is $195 \pm 5$ meV.

In the final analysis, in addition to the bulk SO parameters, the bulk and surface $p_{3 / 2}$ lifetime widths, the bulk singularity index, and the surface-atom core-level shift were constrained to their average values. All other parameters remained free. With these constraints, satisfac- tory fits are obtained to all the data in Fig. 1, yielding the following additional numerical results: The average bulk Gaussian width is $86 \pm 2 \mathrm{meV}$. The average surface Gaussian width is $108 \pm 5 \mathrm{meV}$. (The data indicate some degradation of the resolution of the analyzer at the larger takeoff angles.) The surface SO splitting is smaller than that of the bulk and has a well-defined angular dependence shown in Fig. 4. In order to check whether this behavior is sensitive to the constraints used in the model function, the constrained parameters were varied one at a time. The bulk SO splitting was increased to $257 \mathrm{meV}$, the bulk SO ratio increased to 0.6 , and the surface $3 p_{3 / 2}$ lifetime width assigned values of 30 and $46 \mathrm{meV}$. In each case the quality of the fit was significantly degraded, but the angular dependence of the surface SO splitting remained essentially unchanged, although there were significant changes in the angle-averaged value.

For comparison with the data, we have calculated the centroid shift produced by a crystal-field potential for a bcc(110) atom, following Herbst. ${ }^{13}$ For this twofold geometry only the $Y_{20}$ and $Y_{22}$ components of the crystal field make any contribution to the splitting of the $p$ levels. For our experimental geometry the centroid shift is proportional to the $Y_{20}$ component of the potential, $V_{20}$, and is unaffected by the value of the $Y_{22}$ component, $V_{22}$. In calculating the centroid shift we have included refraction of both the incident light and the outgoing photoelectron by the metal surface, and have ignored any possible photoelectron diffraction. The absence of strong photoelectron diffraction is supported by measured bulk to surface intensity ratios which show no strong modulation due to diffraction either as a function of photon energy ${ }^{20}$ or takeoff angle.

The results of the calculations are shown in Fig. 5. In addition to $V_{20}$ there are two adjustable parameters in

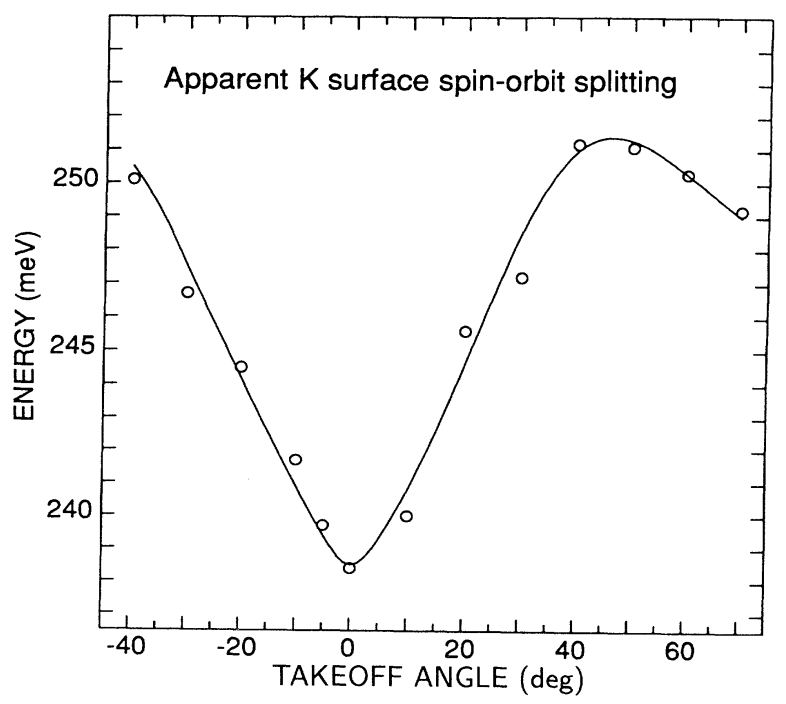

FIG. 4. Takeoff-angle dependence of the apparent surface spin-orbit splitting obtained from the data in Fig. 1. The line is a guide to the eye. 


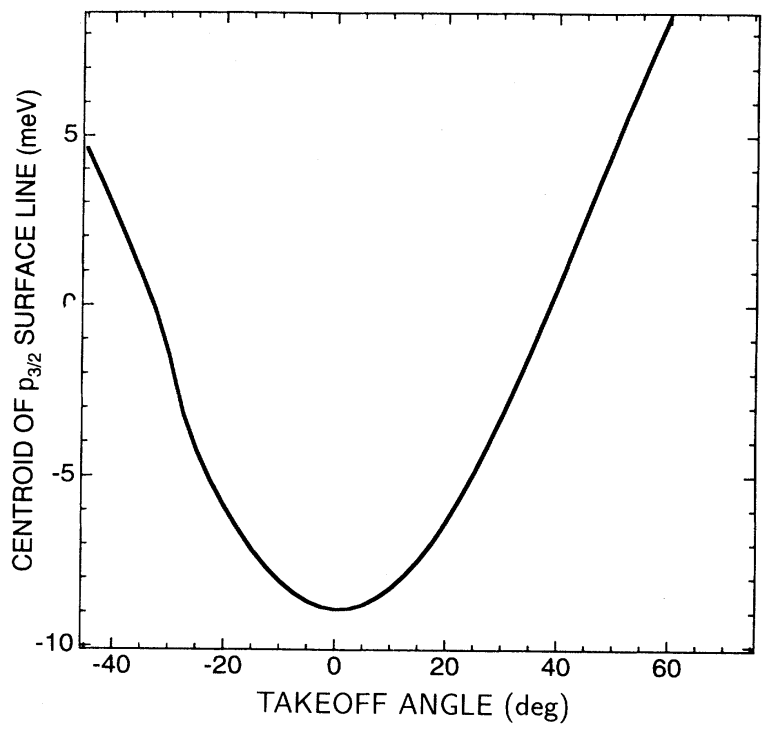

FIG. 5. Calculated takeoff-angle dependence of the centroid shift of the $\mathrm{K} 3 p_{3 / 2}$ crystal-field doublet. The parameters have been adjusted to mimic the data in Fig. 4 (see text).

the calculation: the ratio $R_{d s}$ of the magnitude of $d$-and $s$-wave radial matrix elements, and the relative phase between them. The values of these parameters were adjusted until a minimum at $0^{\circ}$ takeoff angle was obtained. The magnitude of $V_{20}$ was then adjusted so that the magnitude of the centroid shift matched the data shown in Fig. 4. Satisfyingly, the curve is fairly symmetric about $\Theta=0^{\circ}$, although the turnover above $50^{\circ}$ is not reproduced. The curve in Fig. 5 is the result of $R_{d s}=0.5$, $\delta=-10^{\circ}$, and $V_{20}=240 \mathrm{meV} \AA^{-2}$. Although no specific calculation exists for $\mathrm{K}$, calculations of $R_{d s}$ and $\delta$ for $3 p$ photoemission at the same kinetic energy for nearby elements $\mathrm{Ni}, \mathrm{Cu}$, and $\mathrm{Ga}$ all show a value of $R_{d s}$ between 0.3 and 1.0 with a small phase angle $\delta$, suggesting that these parameters are entirely reasonable. ${ }^{21}$

To assess the implications of $V_{20}=240 \mathrm{meV} \AA^{-2}$, we have calculated $V_{20}$ (and also $V_{22}$ ) arising from the first three neighboring-atom shells assuming Thomas-Fermi potentials for the neighbors. The results of that calculation are shown in Fig. 6, where $V_{20}$ and $V_{22}$ are plotted as functions of the Thomas-Fermi screening length $\lambda_{\mathrm{TF}}$. For $\lambda_{\mathrm{TF}}=0.75 \AA$, which results from a free-electron approximation for $\mathrm{K}, V_{20}=13 \mathrm{meV} \AA^{-2}$, a value much smaller than that needed to mimic the data. A value of $240 \mathrm{meV} \AA^{-2}$ is obtained for $\lambda_{\mathrm{TF}}=3.0 \AA$, which although larger than $0.75 \AA$ is still an atomic-scale length. With $V_{20}=240 \mathrm{meV}^{-2}$ and the corresponding $V_{22}=-24 \mathrm{meV} \AA^{-2}$, the calculated crystal-field splitting of the two $p_{3 / 2}$ components is $38 \mathrm{meV}$, much smaller than can be resolved.

The discrepancy in $V_{20}$ may be due to a number of mechanisms. First, the Thomas-Fermi description of the electronic response underestimates the spatial extent of the Fermi-sea screening, since it does not account for the

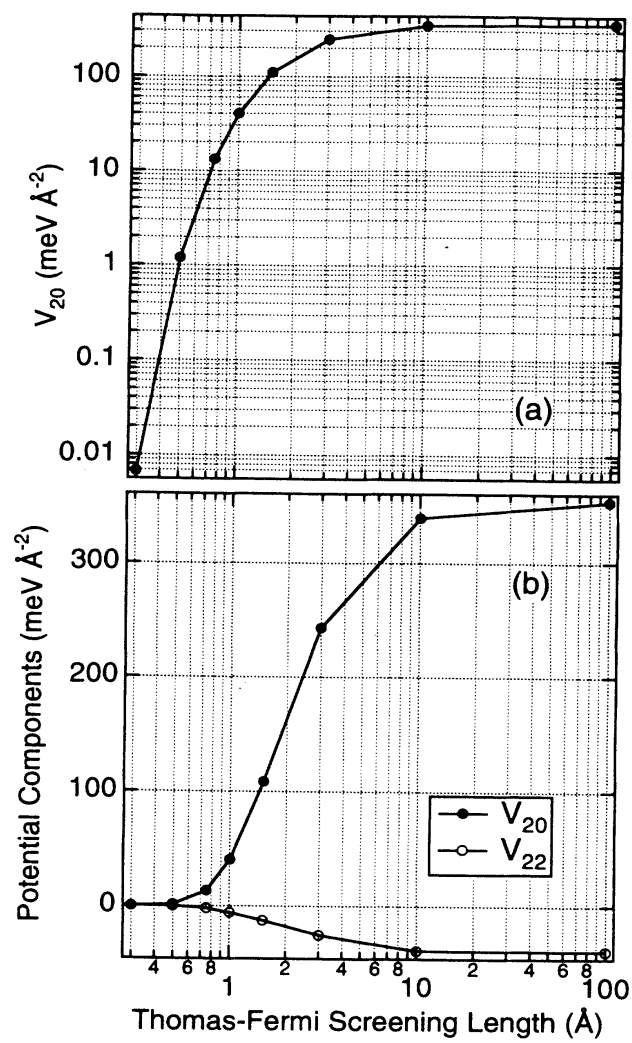

FIG. 6. Crystal-field potential components $V_{20}$ and $V_{22}$ as functions of the Thomas-Fermi screening length.

finite momentum cutoff inherent in having states filled only to the Fermi level. Second, the calculation neglects final-state aspects of the measurements. Screening of the core hole doubles the conduction-electron charge density near the hole-state atom, and may have a major effect on the crystal field. There may also be initial-state contributions from the relaxation of the spacing of the lattice planes near the surface and from the readjustment of the conduction-electron charge at the surface which produces the surface dipole layer. These aspects would have to be investigated before one can conclude that the small size of the calculated shift rules out crystal-field splitting as the source of the observed shift.

These results indicate that the crystal-field splitting is so small compared with the phonon width that it cannot possibly be resolved in photoemission spectra, and, in general, will not even produce significant line broadening. It is sufficiently large, however, to explain why a consistent set of bulk and surface SO parameters for data taken at different takeoff angles could not be found before the possibility of a crystal-field modulation was considered. The findings suggest that the surface SO splitting cannot be reliably determined without investigating its angular dependence, and that small differences between bulk and surface SO splittings in other alkali metals $^{22,23}$ may have contributions from crystal-field splitting. Even the then puzzling finding ${ }^{24}$ that the $\mathrm{W} 4 f_{5 / 2}$ spectrum from $\mathrm{W}(100)$ could not be reproduced by a 
Lorentzian-broadened $4 f_{7 / 2}$ spectrum may have its origin on the crystal field. Data from W(110) show a small difference between the bulk and surface SO splittings. Finally, this perturbation impinges on the determination of the surface-atom core-level shift, which may exhibit a spurious angle dependence if determined from the separation of lines with crystal-field splitting. For measurements based on $d$ or $f$ levels, an accurate determination of the surface-atom core-level shift requires a check of the angular dependence to rule out a possible crystal-field perturbation.

Photoemission research was carried out at the National Synchrotron Light Source (NSLS), Brookhaven National Laboratory, which is supported by the Department of Energy, Division of Materials Sciences and Division of Chemical Sciences.
${ }^{1}$ P. H. Citrin, G. K. Wertheim, and Y. Baer, Phys. Rev. Lett. 41, 1425 (1978); Phys. Rev. B 27, 3160 (1983).

${ }^{2}$ D. M. Riffe, G. K. Wertheim, and P. H. Citrin, Phys. Rev. Lett. 67, 116 (1991).

${ }^{3}$ G. K. Wertheim, D. M. Riffe, and P. H. Citrin, Phys. Rev. B 49, 2277 (1994).

${ }^{4}$ G. K. Wertheim, D. M. Riffe, and P. H. Citrin, Phys. Rev. B 45, 8703 (1992).

${ }^{5}$ D. M. Riffe, G. K. Wertheim, and P. H. Citrin, Phys. Rev. Lett. 63, 1976 (1989).

${ }^{6}$ J. F. van der Veen, P. Heiman, J. F. Himpsel, and D. E. Eastman, Solid State Commun. 37, 555 (1981).

${ }^{7}$ D. M. Riffe and G. K. Wertheim, Phys. Rev. B 47, 6672 (1993).

${ }^{8}$ L. I. Johansson, H. I. P. Johansson, J. N. Andersen, E. Lundgren, and R. Nyholm, Phys. Rev. Lett. 71, 2453 (1993).

${ }^{9}$ B. Johansson and N. Martensson, Phys. Rev. B 21, 4427 (1980).

${ }^{10}$ D. M. Riffe, G. K. Wertheim, D. N. E. Buchanan, and P. H. Citrin, Phys. Rev. B 45, 6216 (1992).

${ }^{11}$ B. J. Waclawski and J. F. Herbst, Phys. Rev. Lett. 35, 1594 (1975).

${ }^{12}$ W. F. Egelhoff, Jr., D. L. Perry, and J. W. Linnett, Surf. Sci. 54, 670 (1976).

13J. F. Herbst, Phys. Rev. B 15, 3720 (1977).
${ }^{14} \mathrm{The}$ growth of $\mathrm{K}$ on $\mathrm{Ni}(100)$ is similar to that of $\mathrm{Na}$ reported by S. Andersson, J. B. Pendry, and P. M. Echenique, Surf. Sci. 65, 539 (1977).

${ }^{15}$ G. K. Wertheim and D. M. Riffe, Solid State Commun. (to be published).

${ }^{16}$ G. K. Wertheim, J. Electron Spectrosc. Relat. Phenom. 60, 237 (1992)

${ }^{17}$ S. Doniach and M. Šunjić, J. Phys. C 3, 285 (1970).

${ }^{18}$ M. W. D. Mansfield, Proc. R. Soc. London Ser. A 346, 539 (1975).

${ }^{19}$ R. L. Fink, P. N. First, and C. P. Flynn, Phys. Rev. B 38, 5839 (1988).

${ }^{20}$ G. K. Wertheim, D. M. Riffe, N. V. Smith, and P. H. Citrin, Phys. Rev. B 46, 1955 (1992).

${ }^{21}$ S. M. Goldberg, C. S. Fadley, and S. Kono, J. Electron Spectrosc. Relat. Phenom. 21, 285 (1981).

${ }^{22}$ G. K. Wertheim, D. N. E. Buchanan, and J. E. Rowe, Solid State Commun. 77, 903 (1991).

${ }^{23}$ G. K. Wertheim and D. N. E. Buchanan, Phys. Rev. B 43, 13815 (1991).

${ }^{24}$ G. K. Wertheim, P. H. Citrin, and J. F. van der Veen, Phys. Rev. B 30, 4343 (1984). 